\title{
DESARROLLO DE UN SISTEMA DE SUPERVISIÓN DE VARIABLES DE FUNCIONAMIENTO PARA LA PLANTA TÉRMICA DE LA FUNDACIÓN UNIVERSIDAD DE AMÉRICA
}

DEVELOPMENT OF A MONITORING SYSTEM FOR OPERATING VARIABLES OF THE THERMAL POWER PLANT

OF THE UNIVERSITY OF AMERICA FOUNDATION

Sebastián López Agudelo*

José Luis Morales Gómez**

Miguel Alfonso Morales Granados†

Carlos Urrego Rodríguezł

FUNDACIÓN UNIVERSIDAD DE AMÉRICA

Recibido: 2 de septiembre de $2018 \quad$ Aceptado: 24 de junio de 2019

DOI: https://doi.org/10.29097/23461098.311

\section{Resumen}

Este proyecto desarrolló la programación de una pantalla que funciona como herramienta de monitoreo para la planta térmica adquirida por la Universidad. Apoyado por LabVieW, el aplicativo se fundamentó en la instrumentación de la planta con el fin de analizar los datos obtenidos por los sensores. Para el funcionamiento de esta herramienta fue necesario caracterizar los componentes de la planta; así se entendió cómo opera y qué propiedades de comunicación incluye, asimismo se realizó el plano P\&ID, en el que se identificaron los lazos de control. Con estos parámetros se diseñó un software interactivo, que, a través de una interfaz de usuario, informa al operario sobre el estado de los equipos, las alarmas, los datos en tiempo real, la adquisición de estos, su historial y las eventualidades en los aparatos. Las similitudes con el sistema SCADA podrán aplicarse en investigación como complemento del conocimiento práctico en las materias afines.

Palabras clave: programación, LabVieW, supervisión, SCADA, interfaz, monitoreo.

\section{Abstract}

This project developed the programming of a screen that works as a monitoring tool for the thermal plant acquired by the University. Using LabView, this application was based on the instrumentation of the plant to analyze the data obtained by the sensors. For operating this tool, it was necessary to characterize the components of the plant, thus it was understood how it works and the communication properties it includes. It was also made the P \& ID plan, in which the control loops were identified. With these parameters,

\footnotetext{
* Ingeniero mecánico. Coinvestigador Grupo de Investigación en Térmicas y Fluidos, GITEF.

** Ingeniero mecánico. Coinvestigador Grupo de Investigación en Térmicas y Fluidos, GITEF.

† Ingeniero mecánico. Coinvestigador Grupo de Investigación en Térmicas y Fluidos, GITEF.

凶. miguel.morales@profesores.uamerica.edu.co

‡ Ingeniero mecánico. Coinvestigador Grupo de Investigación en Térmicas y Fluidos, GITEF.
} 
the interactive software was designed, which, through a user interface, informs the operator about the condition of the equipment, alarms, real-time data, data acquisition, history, and eventualities. Similarities to the SCADA system can be applied in research as a complement to practical knowledge in related areas.

Keywords: programming, LabView, supervision, SCADA, interface, monitoring.

\section{INTRODUCCIÓN}

En la actualidad, los procesos industriales se enfocan en una menor participación humana con el fin de disminuir errores de operación. Aprovechando los instrumentos disponibles en la reciente adquisición de una planta térmica parcialmente automatizada por parte de la Fundación Universidad de América, nació la posibilidad de desplegar las herramientas existentes para llevar a cabo una investigación de los fenómenos que ocurren durante el funcionamiento de dicha planta, con la adquisición y análisis de los datos obtenidos en el PLC que la dirige. Este articulo presenta el desarrollo de un sistema de supervisión de variables de funcionamiento que, con los instrumentos instalados, propone tres interfaces para el monitoreo de la planta térmica, fundamentado en el modelo SCADA y programado con el software LabView.

\section{METODOLOGÍA}

El sistema de supervisión de variables de funcionamiento de la planta térmica se desarrolló a través de las siguientes tareas:

- Caracterización de los instrumentos instalados en la planta térmica.

- Identificación de lazos de control y elaboración del plano P\&ID.

- Desarrollo de programación de una interfaz de usuario.

\section{Caracterización de los instrumentos}

La planta térmica adquirida por la Universidad de América cuenta con diferentes tipos de instrumentos que permiten el control en las operaciones de trabajo. Para garantizar la compatibilidad de la interfaz con el sistema por controlar y la correcta identificación de los lazos de control, fue necesario obtener información sobre, la información de operatividad, las formas de comunicación de datos y el tipo de control. Se realizó una serie de visitas con el fin de identificar cada uno de los instrumentos que intervienen en el funcionamiento. En la tabla 1 se pormenorizan las cantidades encontradas.

\section{Tabla 1}

Caracterización de los instrumentos

\begin{tabular}{lll}
\hline Tipo de instrumento & Cantidad & Descripción \\
\hline Sensores & 17 & Presostato Honeywell ${ }^{\mathrm{a}}$ L404F (1)
\end{tabular}


Desarrollo de un sistema de supervisión de variables

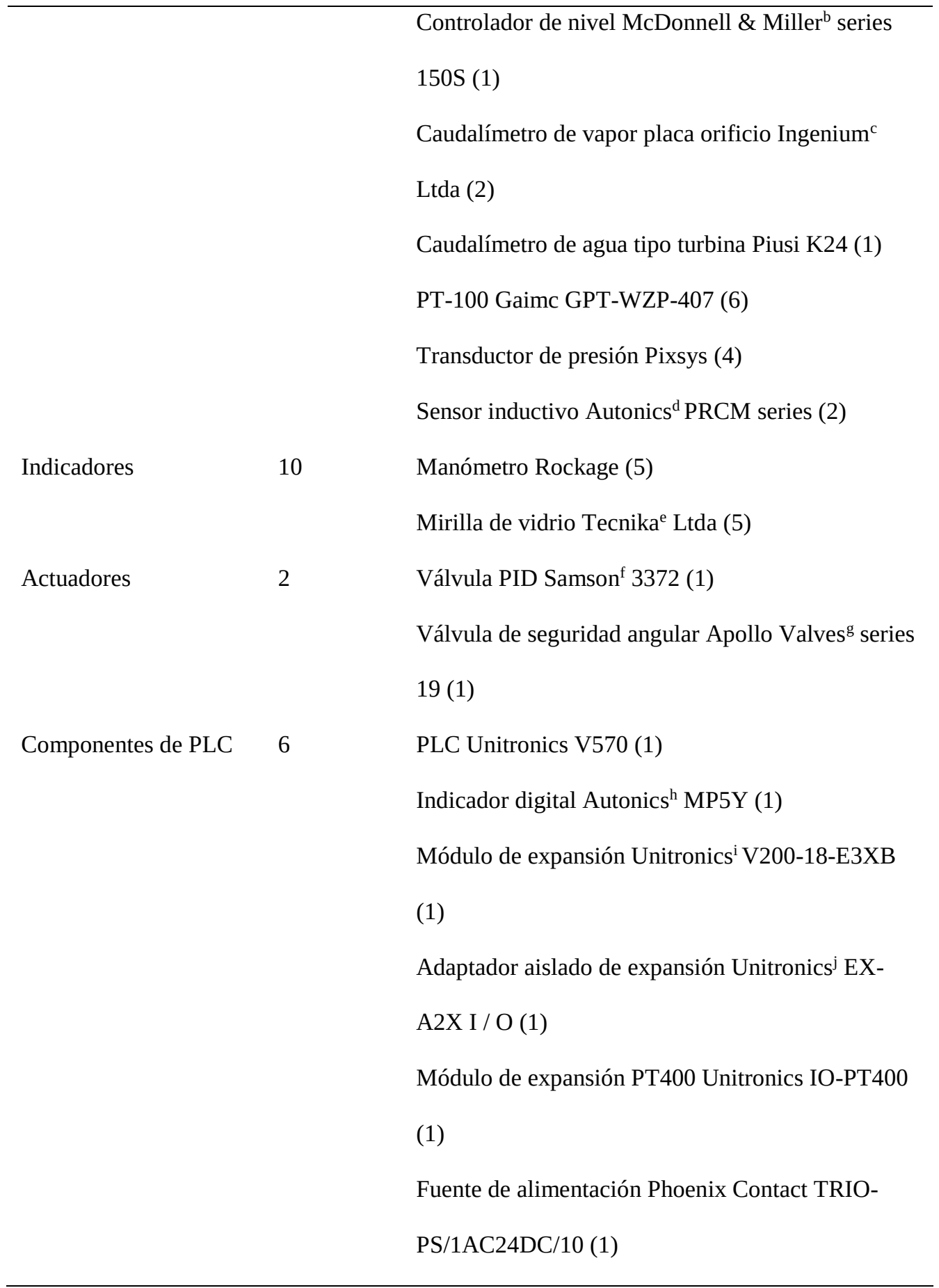

Nota. a Honeywell International (2004); b McDonnell \& Miller (2008); c Ingenium (2018); d Autonics (s. f.); e Tecnika (s. f.); f Samson (2004); g Apollo Valves (2019); h Autonics, (s. f.); i Unitronics (2006); j Unitronics (2009). Tabla elaboración propia basada en fichas técnicas de los fabricantes de los productos (Ingenium. 2018). 
López Agudelo, Morales Gómez, Morales Granados y Urrego Rodríguez

\section{Identificación de lazos de control y elaboración del plano P\&ID}

El entendimiento de los procesos de funcionamiento de la planta procuró identificar los lazos de control y el orden en que ocurren. Con la caracterización de los instrumentos se logró una correcta identificación de estos lazos y la elaboración del plano P\&ID, pertinente para la planta térmica, siguiendo los lineamientos de la Norma ISA. (Instrument Society of America, ISA, 1992)

Aparte de contarse como requisito para el desarrollo de la interfaz de usuario, el plano P\&ID facilita las actividades de mantenimiento, ya que describe el flujo del proceso e identifica cada uno de los componentes presentes en la planta térmica.

Se identificaron siete lazos de control. El orden descriptivo corresponde al ciclo de funcionamiento de la planta.

- $\quad$ Lazo de control 1. Alimentación de ACPM a quemador de caldera.

- $\quad$ Lazo de control 2. Alimentación de gas natural a quemador de caldera.

- Lazo de control 3. Alimentación de agua a caldera.

- $\quad$ Lazo de control 4. Salida de vapor y distribución.

- Lazo de control 5. Funcionamiento de ciclo térmico y generación de energía.

- $\quad$ Lazo de control 6. Funcionamiento de suavizador de agua.

- $\quad$ Lazo de control 7. Funcionamiento de intercambiadores de calor.

\section{Selección del software de programación}

Para esta se usó la ponderación lineal o scoring como método de decisión. Este ayuda a la toma de decisiones en situaciones de poca información de modo sencillo y rápido, e identifica la opción más favorable en una decisión multicriterio (Pacheco y Contreras, 2008, p. 37). Tras la aplicación del método se obtuvo la siguiente tabla, donde se identificaron las alternativas, criterios de selección, la ponderación de cada uno de los criterios y el cálculo del score.

Tabla 2

Selección de software mediante scoring

\begin{tabular}{cccccc}
\hline Criterios: & Ponderación & LabView & LaBwindows & HP-VEE & UniLogic \\
\hline Simulación & 5 & 9 & 8 & 8 & 5 \\
Tipo de & 4 & 7 & 5 & 5 & 7 \\
programación & & & & & \\
Programas .EXE & 3 & 8 & 7 & 1 & 6 \\
\hline
\end{tabular}




\begin{tabular}{|c|c|c|c|c|c|}
\hline $\begin{array}{l}\text { Ampliación de } \\
\text { librerías externas }\end{array}$ & 3 & 8 & 9 & 8 & 1 \\
\hline $\begin{array}{c}\text { Comunicación } \\
\text { con el PLC }\end{array}$ & 5 & 8 & 7 & 7 & 9 \\
\hline $\begin{array}{c}\text { Aplicación } \\
\text { básica de un } \\
\text { sistema SCADA }\end{array}$ & 4 & 9 & 8 & 7 & 9 \\
\hline Total, & & 197 & 175 & 150 & 155 \\
\hline
\end{tabular}

Nota. Tabla de elaboración propia.

Según los criterios expuestos en el método scoring, se seleccionó LabView, software de ingeniería especializado en la informática industrial y científica basado en código gráfico (National Instruments, 2018).

\section{Programación de la interfaz}

El desarrollo de la interfaz se fundamentó en el funcionamiento de la planta con el ciclo de Rankine recalentado, ciclo termodinámico donde un fluido, generalmente agua, se calienta hasta su temperatura de ebullición por medio de una caldera alimentada por combustibles fósiles como carbón o gas; luego, el vapor se lleva a una turbina y genera energía; por último, este fluido retoma sus características iniciales para repetir el proceso hasta suplir la demanda energética (Patiño y Oliveros, 2011). El proceso se ilustró interactivamente para uso académico, dividiéndolo en los principales lazos de control anteriormente identificados.

Además, este software cuenta con características usadas en la industria para la comunicación hombre-máquina, como el sistema SCADA (Supervisory Control And Data Acquisition), que traduce control supervisor y adquisición de datos. Se concibe como una herramienta que permite el acceso a la información de planta desde un punto remoto (Rodríguez Penin, 2007, p. xiii).

Se identifica un SCADA por las siguientes prestaciones (Zolotová y Landryová, 2000):

- Monitorización

- Supervisión

- Adquisición de datos 
- Alarmado de proceso

- Pantallas de información

- Almacenamiento de la información y reportes

- $\quad$ Secuencia de adquisición de eventos

El medio por el cual el operario interactúa con la maquina tiene por nombre HMI (Human Machine Interface). Se trata de una interfaz que hace parte de un sistema informático, cuya función es interactuar directamente con la máquina (Copadata, 2018). En ISO 9241-110, se define interfaz como "todos los componentes de un sistema interactivo que suministra información y controles para que el usuario pueda realizar tareas específicas con el sistema interactivo”. El entorno del HMI se compone de tres pantallas:

- Pantalla 1: caldera y tanque de condensados

- Pantalla 2: generación de energía, condensador y torre de enfriamiento

- Pantalla 3: intercambiadores de calor y abastecimiento del distribuidor de vapor

En las pantallas se incluyeron los equipos, instrumentos, válvulas y tubería de la planta que se representaron con gráficas, elementos configurados como booleanos de manera que cambiaran de color cuando se presentara una alteración de estado o apagado/encendido. Del mismo modo, los instrumentos adquisidores de datos se dispusieron como indicadores para mostrar al operario el valor medido. Además, cada pantalla contó con un tutorial de funcionamiento en el que se explicaban los pasos para conocer cada interfaz por completo.

\section{Figura 1}

Representaciones gráficas

Suavizador de agua
SA-001
Encendido
Suavizador de agua
SA-001




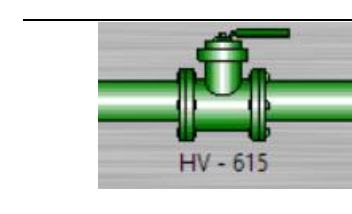

Con fluido

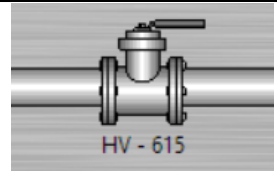

Sin fluido

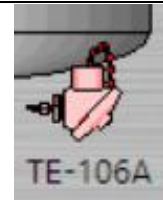

Encendido

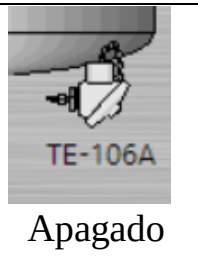

Apagado

Nota. Esquema de elaboración propia.

El sistema de alarmas cuenta con tres tipos de indicadores. El primero determina el estado actual del equipo, representando con azul claro su funcionamiento normal, mientras que el color naranja indica advertencia o una posible falla en el equipo. El segundo tipo se encuentra específicamente en los tanques de valor mínimo y valor máximo de nivel. El tercero, en ventanas emergentes que avisan al usuario las acciones para normalizar el funcionamiento del software.

\section{Figura 2}

Tipos de nivel alto

Nivel alto tipo 1

Nivel alto tipo 3 


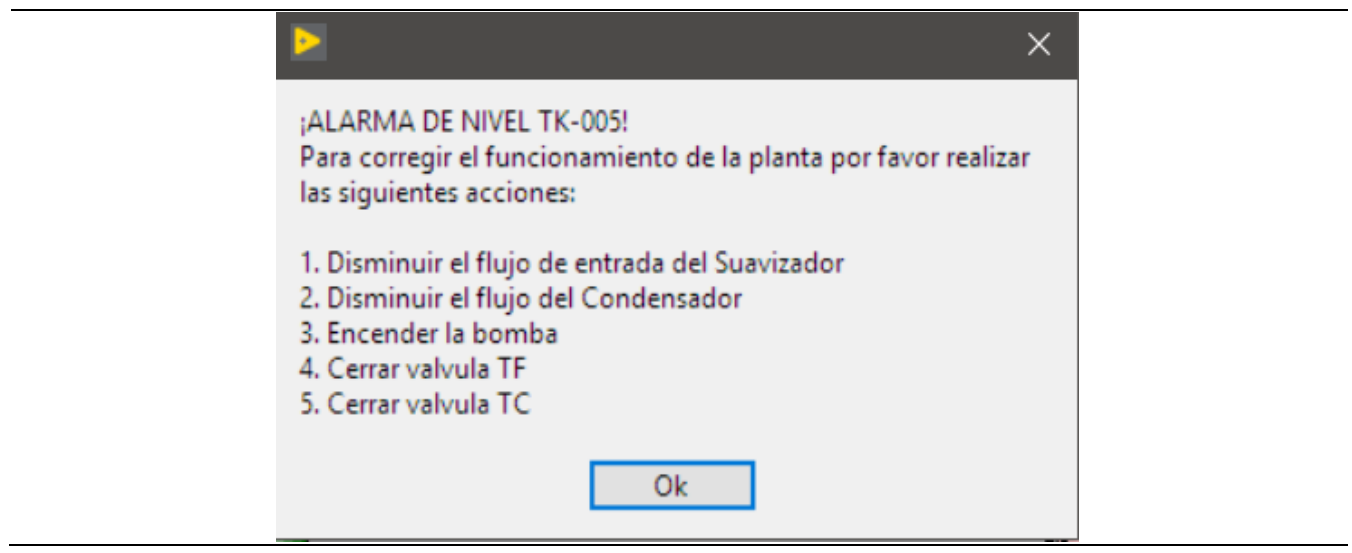

Nota. Esquema de elaboración propia.

Dentro de las pantallas se encuentra el "centro de operación”, panel en el que el usuario puede acceder a la base de datos obtenida por el software, ver graficas de funcionamiento y generar reportes desde el arranque, que cuentan con un historial de datos de los instrumentos de cada pantalla. Consecuentemente, incluye antecedentes de alarma de los equipos y una gráfica expositora con los datos de los instrumentos.

\section{Figura 3}

Reporte y gráfica de comportamiento A

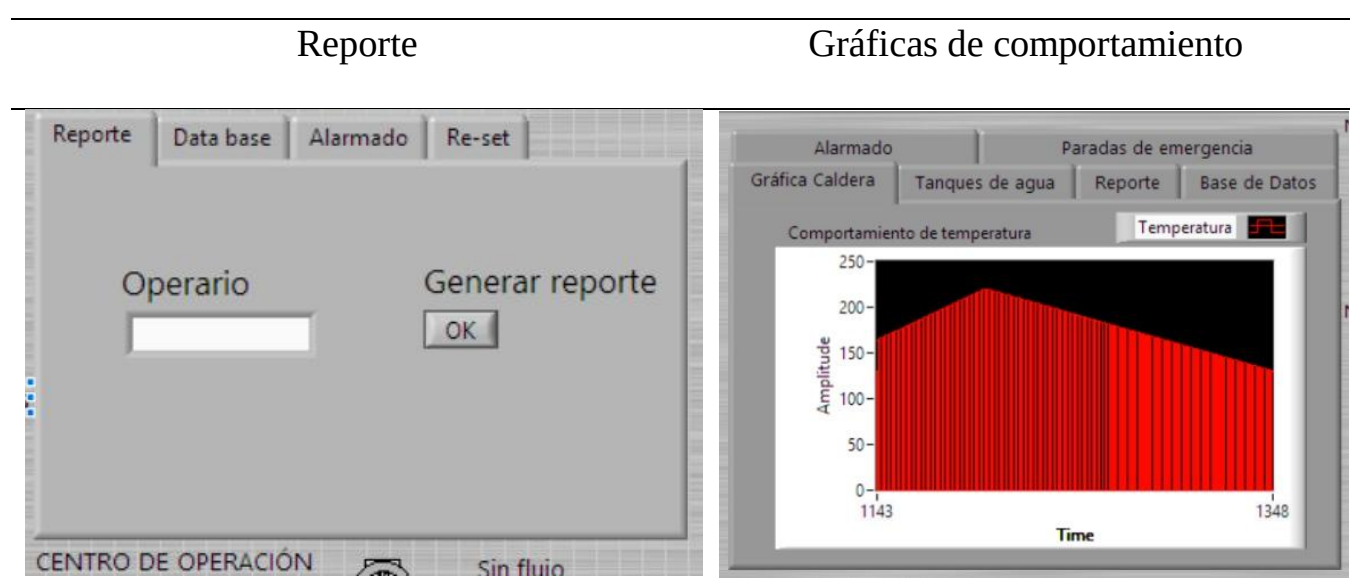

Nota. Esquema de elaboración propia. 
Figura 4

Reporte y gráficas de comportamiento $B$

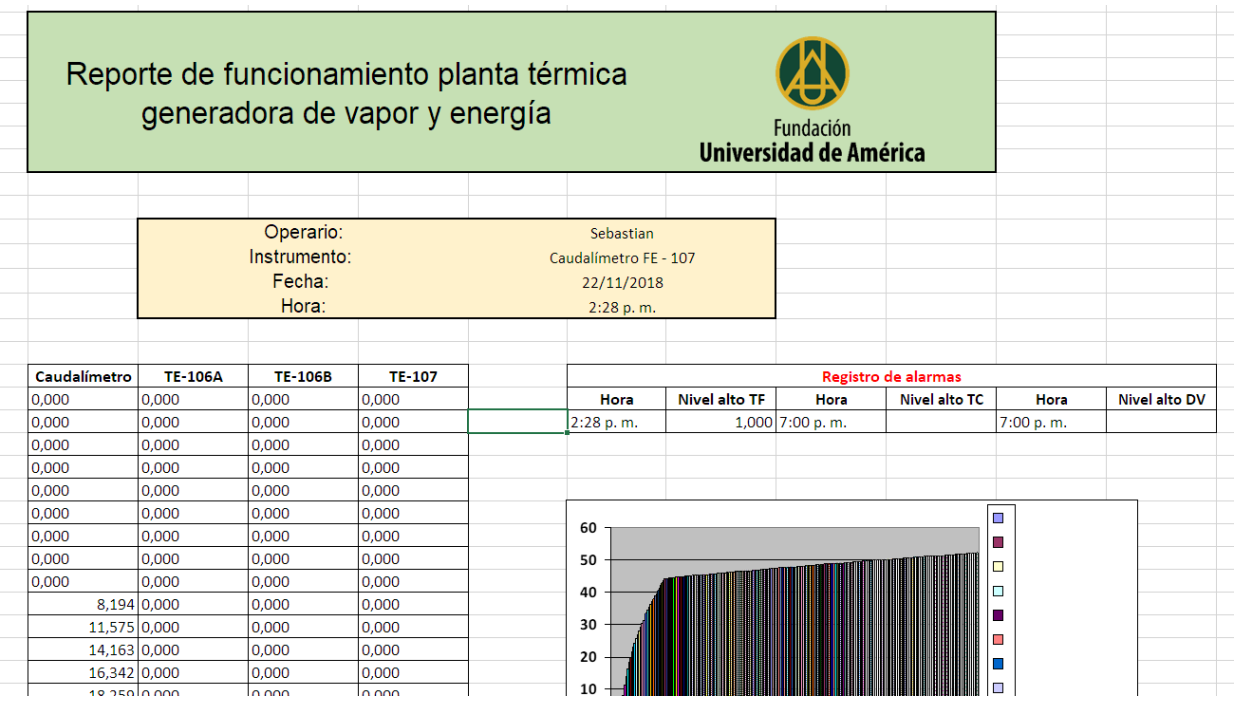

Nota. Esquema de elaboración propia.

Como resultado del software se obtuvieron las siguientes pantallas, representadas con las figuras 5, 6 y 7, en las que se distribuyeron los componentes de la planta.

\section{Figura 5}

\section{Pantalla N.ㅇ 1 de caldera}

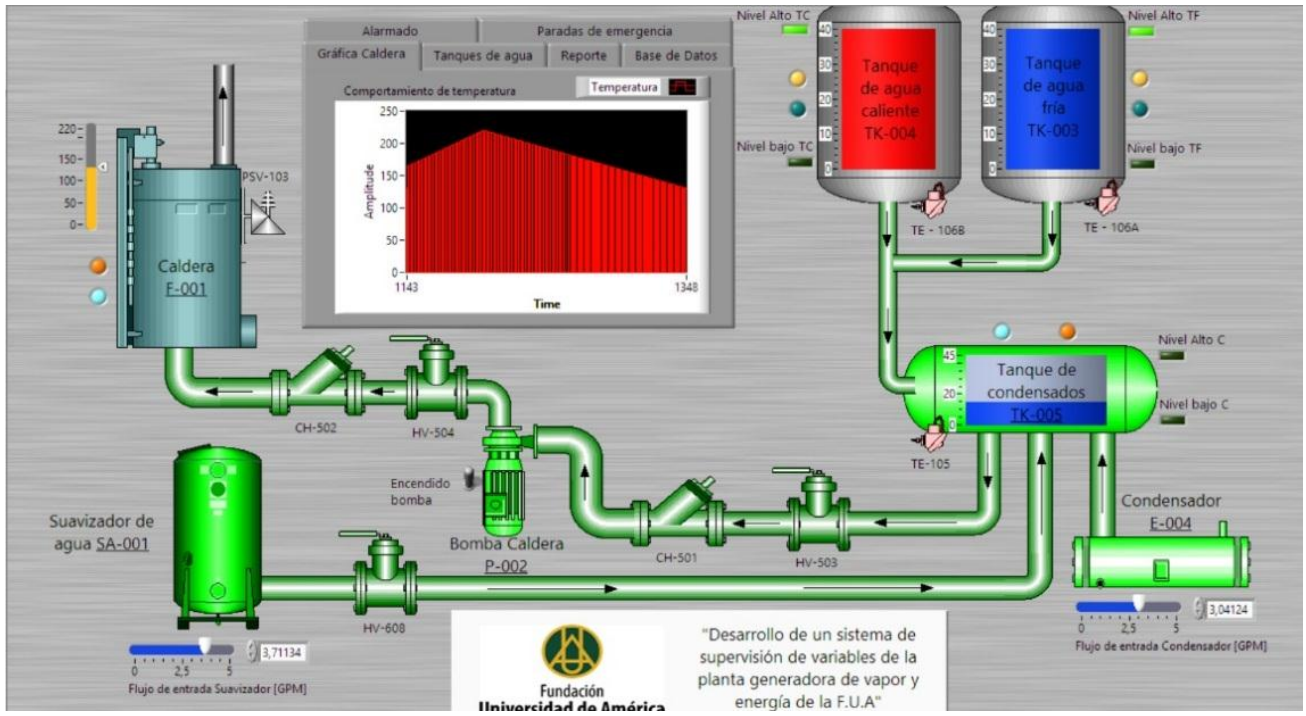

Nota. En la gráfica se muestra: caldera, adquisición de datos, flujo de entrada suavizador y condensador, bomba de caldera, nivel alto de equipos y tanque de condensados. 
En esta interfaz se relacionaron los subprocesos de alimentación de agua a la caldera, suministro de agua a los tanques de agua fría y caliente, funcionamiento del condensador, suavizador de agua y entradas del tanque de condensado; como característica especial presenta la gráfica de comportamiento de la caldera, que permite al usuario verificar el funcionamiento normal de operación.

\section{Figura 6}

Generador de energía

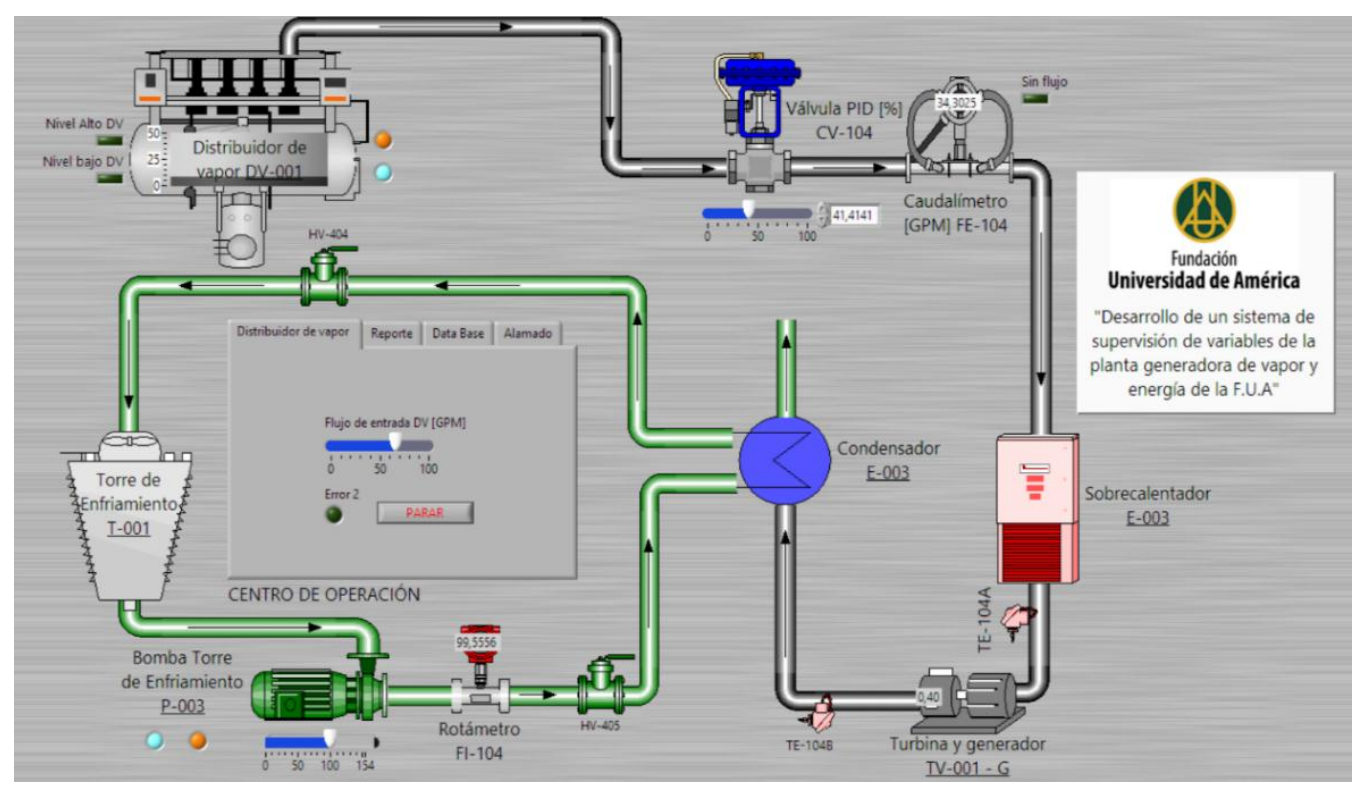

Nota. En la gráfica se muestra: condensador, válvula PID, caudalímetro, sobrecalentador, turbina y generador, torre de enfriamiento y centro de operaciones.

Esta interfaz presenta los procesos de generación de energía y de funcionamiento del distribuidor de vapor; considerados los más importantes de la planta. Además, muestra el ciclo de enfriamiento de agua a la salida del generador — de gran significado-, ya que, para retornar al tanque de condensados, el fluido del proceso debe encontrarse en estado líquido.

La interfaz de los intercambiadores de calor completa la demostración del funcionamiento de todos los lazos de control identificados en la planta térmica, obteniendo así el flujo de alimentación del tanque de agua caliente, que depende del cambio de calor de los dos equipos instalados. Además, especifica que la alimentación del tanque de agua fría proviene del suavizador de agua, con esto se justifica que los drenajes del intercambiador y suavizador alimente directamente el tanque de condensado presentado como pantalla en la figura 5. 


\section{Figura 7}

Intercambiadores de calor y abastecimiento del distribuidor de vapor

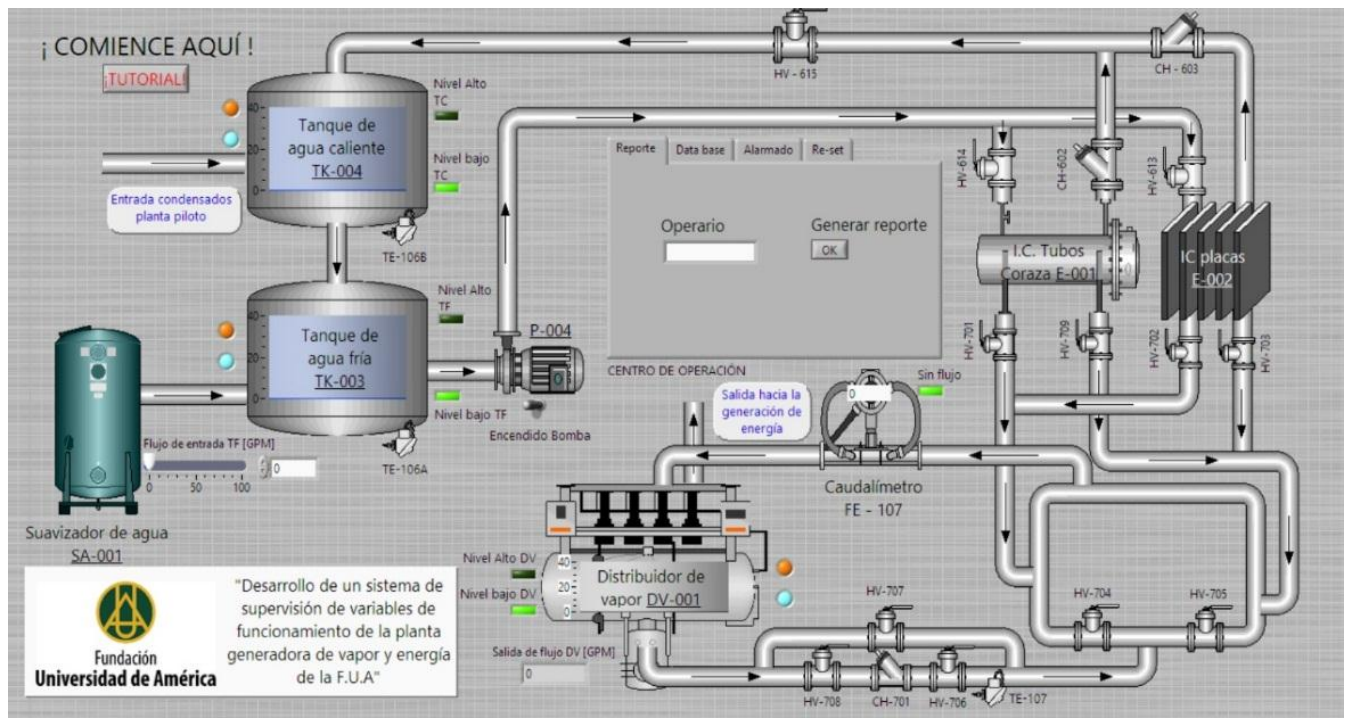

Nota. Esquema de elaboración propia.

Cada una de estas pantallas cuenta con el tag de identificación relacionado en el plano P\&ID y elaborado según el artículo "Metodología para la estandarización de componentes SCADA bajo la Norma ISA” de la revista Visión Electrónica, que destaca los beneficios de aplicar esta norma en los procesos de automatización gracias al mejoramiento de la calidad, a las alertas tempranas, mejor tiempo de respuesta, aumento de la efectividad del operador, seguridad de manejo de información y procesos, flexibilidad, facilidad procedimental, y estandarización (Donado y Villamizar, 2018).

\section{ANÁLISIS DE RESULTADOS}

Durante el desarrollo del software de supervisión de variables, se encontró que en la industria de la automatización existen sistemas como el SCADA, que cumplen con los lineamientos necesarios para aplicarse a este proyecto. Con base en los componentes y objetivos de este sistema se obtuvo un software académico con la capacidad de ejercer monitoreo de la planta.

No solo se trata de una alternativa gráfica fácil de usar, sino también de un complejo sistema de adquisición y almacenamiento de datos (en tiempo real, histórico), monitoreo, supervisión y con indicadores para respaldar decisiones. Igualmente, el modelo SCADA destaca por su capacidad de conexión y comunicación con niveles tecnológicos e informativos más altos. Con su aplicación la visualización del proceso es diferente frente a otras (Sobota, 1998).

Debido a que fue imposible conectar el software con la planta térmica, se vislumbró la posibilidad de simular los datos y comportamiento de los equipos; esto consiguió que 
se puedan estudiar diferentes configuraciones de simulación sin necesidad de arriesgar los equipos instalados, y así comprobar el buen funcionamiento de la planta al operar.

\section{CONCLUSIONES}

Al lograr la comprensión de las características de cada uno de los instrumentos instalados en la planta, se identificó su manera correcta de funcionamiento, rangos de operación, las directrices necesarias para modificar y controlar variables, y las facilidades de comunicación de datos. Con esto se reconocieron los lazos de control y la forma de usar los datos para desarrollar las herramientas contempladas en este proyecto.

El funcionamiento de la planta, los lazos de control con los elementos que la componen y la identificación de la instrumentación instalada, mostrada en el plano P\&ID, facilitaron la determinación de los procesos y las actividades de mantenimiento.

Del sistema de supervisión de variables se obtiene una herramienta de gestión para la planta térmica, de forma que permite al usuario, de modo simple y didáctico, entender en tiempo real el funcionamiento del equipo, las alarmas de instrumentos, la adquisición de datos, su historial y los reportes desde el momento de encendido, brindándole los elementos primarios de un sistema SCADA. Esta información podrá basar investigaciones, extenderse a materias afines y al control de datos.

\section{Referencias}

Apollo Valves. (2019). 19-Series Bronze Safety Valves for Steam, Air \& Gas Service. http://www.epmc.co.th/Safety\%20Valves/pdfs/Apollo\%20\%20Conbraco\%2019\%20Series\%20Safety\%20Valve.pdf

Autonics. (s. f.). MP5S/MP5Y/MP5W/MP5M Series. https://www.autonics.se/produkt/mp5s-mp5y-mp5wseries/\#: :text=High\%20performance\%20digital\%20pulse\%20meters,measu rements\%20and\%20feature\%20various\%20functions.

Autonics. (s. f.). Sensores de proximidad. Autonics. http://dominion.com.mx/descargas/sensores-de-proximidad.pdf

Copadata. (2018). ¿Qué es una HMI? Copadata. https://www.copadata.com/es/soluciones-hmi-scada/interfaz-hombremaquina-hmi/

Donado y Villamizar (2018). Visión Electrónica (2017). Metodología para estandarización de componentes SCADA bajo normas ISA. https://revistas.udistrital.edu.co/index.php/visele/article/view/13402/13869

Honeywell International. (2004). L404F,T,V. PressureTrol Controllers. Honeywell. http:/lit.stromquist.com/docs/HONEYWELL/REGULAR/71-2429.PDF

Ingenium. (2018). Planta térmica didáctica. (Fundación Universidad de América.)

Instrument Society of America, ISA. (1992). Instrumentation Symbols and Identification. ISA. 
ISO 9241-110 (International Organization for Standarization) Ergonomics of humansystem interaction. (2006). https://www.iso.org/standard/38009.html

McDonnell \& Miller. (2008). Series 150S and 157S Low Water Cut-Off/Pump Controllers, Installation and Maintenance Instructions. ITT Corporation. https://dominion.com.mx/fichas/control-de-nivel-mcdonnell-miller-259.pdf

National Instruments. (2018) ¿Qué es LabView? National Instruments. http://www.ni.com/es-co/shop/LabView.html

Pacheco, F., y Contreras, E. (2008). Manual metodológico de evaluación multicriterio para programas y proyectos. Naciones Unidas, Comisión Económica para América Latina y el Caribe, CEPAL. https://www.dii.uchile.cl/wpcontent/uploads/2011/06/manual58-Ilpes.pdf

Patiño Pérez, C. F., y Oliveros Bayona, P. J. (2011). Instrumentación de la planta térmica de la Universidad

Pontificia Bolivariana. (Trabajo de grado). Universidad Pontificia Bolivariana. https://repository.upb.edu.co/handle/20.500.11912/1683

Redrejo, J. L. [2008]. Desarrollo de sistemas de regulación y control. http://www.infoplc.net/files/documentacion/control_procesos/infoPLC_net_ ControlPID.pdf

Rodríguez Penin, A. (2007). Sistemas SCADA. Marcombo.

Samson. (2004). Pneumatic and Electropneumatic Actuator Type 3372. Samson Group. https://www.samsongroup.com/document/e83130en.pdf

Tecnika Ltda. (s. f.) Cálculo longitud tubo de vidrio nivel.

Unitronics. (2006). V200-10-E3XB Snap-in I/O Module. (Catálogo). Unitronics. https://www.unitronicsplc.com/wp-content/uploads/2015/12/V200-18E3XBW SPEC 11-06.pdf

Unitronics. (2009). EX.-A2X I/O Expansion Module Adapter, Isolated. (Catálogo). Unitronics.

https://www.i4automation.co.uk/unitronicspdf/EX-A2X_SPEC_12-09.pdf.

Sobota,B. and ValigurskY,M. (1998) Real Time Visualising Kernel for Virtual Reality Application, In: 32nd Spring International Conference, Modelling and Simulation of Systems MOSIS '98. Bystrice p. Host\}'nem, 5.-7.5. 1998, yoU. pp. 111- 116, ISBN 80-75988-23-2.

Zolotová y Landryová, (2000). SCADA/HMI Systems and Emerging Technologies. https://www.sciencedirect.com/science/article/pii/S1474667017355799

Zolotova, l. and Landryova, L. (1999): From Classical SCADAIHMI Systems to Intelligent Component CIM. Virtual Conference Quo Vadis Information Technology, www.xcontrol.sk/konferencia (in Slovak). 
López Agudelo, Morales Gómez, Morales Granados y Urrego Rodríguez

Bibliografía

Acedo Sánchez, J. (2003). Control avanzado de procesos: Teoría y práctica. Díaz de Santos.

Aguilar, E. (2015). Fundamentos de control automático. Instituto Universitario Politécnico Santiago Mariño.

https://es.slideshare.net/EfrainAguilar2/elementos-de-un-sistema-de-controlimportancia-y-comceptos

Amaya, E., y Goitia, A. (1997). Instrumentación industrial. PCI Entrenamiento S. A. https://pdfslide.net/documents/instrumentacion-industrial-55c098d236fd4.html

Armesto Quiroga, J. I. (2008). “Instalación de sistemas de automatización y datos”. (Diapositivas). https://tv.uvigo.es/uploads/material/Video/1452/ISAD Tema4.pdf

Creus Solé, A. (2010). Instrumentación industrial. Marcombo.

Dunn, W. (2005). Fundamentals of Industrial Instrumentation and Process Control. McGrawHill.

Electrónica Fácil. (2004-2020). Fuentes de alimentación. Electrónica Fácil. https://www.electronicafacil.net/tutoriales/Fuentes-alimentacion.php

García Gutiérrez, L. (2018). Teoría de la medición de caudales y volúmenes de agua e instrumental necesario disponible en el mercado. http://www.igme.es/igme/publica/libros2_TH/art2/pdf/teoria.pdf

G-Flow. (2018). Caudalímetros de turbina. G-Flow. http://www.gflow.com/caudalimetros-turbina

Hydrosaar Hydac International. (2008). Sistemas de medición. Hydrosaar. http://www.hydrosaar.com/es/tecnologias/sistemas-de-medicion.html

Instituto Colombiano de Normas Técnicas y Certificación. ICONTEC. (1998). Norma Técnica Colombiana 4490. ICONTEC.

https://www.politecnicojic.edu.co/images/downloads/biblioteca/guias/NTC449 $\underline{0 . p d f}$

Instituto Colombiano de Normas Técnicas y Certificación. ICONTEC. (2008a). Norma Técnica Colombiana 1846. ICONTEC.

http://aprendeenlinea.udea.edu.co/lms/moodle/pluginfile.php/146334/mod_res ource/content/0/Norma Tecnica_Colombiana_NTC 1486 completa archivo.p df

Instituto Colombiano de Normas Técnicas y Certificación. ICONTEC. (2008b). Norma Técnica Colombiana 5613. ICONTEC. 
https://www.politecnicojic.edu.co/images/downloads/biblioteca/guias/NTC561 $\underline{3 . p d f}$

Instrumentation Toolbox. (2013). Basics of the Orifice Plate Flow Meter. https://www.instrumentationtoolbox.com/2013/03/basics-of-orifice-flow-

meter.html

Mendiburu Díaz, H. A. (2003). Automatización medioambiental. Indecopi. https://www.academia.edu/8273992/Automatizacion_Medioambiental?auto=d ownload

Omega. [2018]. La importancia de la instrumentación. https://cl.omega.com/prodinfo/instrumentacion.html

Pinto, L. (2018) Monitoreo y control de procesos. Electroindustria, abril 2006. http://www.emb.cl/electroindustria/articulo.mvc?xid=443\&tip=7

Rodríguez, C. Sistemas de control: elementos componentes, variables, función de transferencia $\quad \mathrm{y}$ diagrama funcional. http://iespoetaclaudio.centros.educa.jcyl.es/sitio/upload/Sistemas de control.p $\underline{\mathrm{df}}$

Smar. [2018]. Medición de presión: Características, tecnologías y tendencias. http://www.smar.com/espanol/ Web

site. http://www.smar.com/espanol/articulos-tecnicos/medicion-de-presioncaracteristicas-tecnologias-y-tendencias.

Wika. [2020]. ¿Cómo funcionan los manómetros mecánicos? https://www.bloginstrumentacion.com/instrumentacion/construccinfuncionamiento-de-manmetros-mecnicos/

Xenics. Process Monitoring \& Quality Control. http://www.xenics.com/en/application/process-monitoring-quality-control 